\title{
EVALUATION OF COLLAGENATED CORTICO-CANCELLOUS XENOGRAFT PLUS LEUKOCYTE-PLATELET RICH FIBRIN AS A GRAFT MATERIAL PRIOR TO DENTAL IMPLANTATION (COMPARATIVE CLINICAL, RADIOGRAPHICAL \& HISTOLOGICAL STUDY)
}

\author{
Tamer Nabil Guirguis Ishak ${ }^{*}$; Laila Mostafa Omara*; \\ Hassan Abd-ElGhany Osman ${ }^{* * *}$ and Reham Mohamed Hamdy ${ }^{* * * *}$
}

\begin{abstract}
The dimensions of the alveolar bone are of great importance from both esthetic and implant planning reasons. The preservation of bone volume immediately after tooth removal might be necessary to optimize the success of implant placement in terms of esthetics and function. This study was conducted to investigate histologically, clinically \& radiographically, the potential of combination of xenogenic cortico - cancellous bone graft and platelet Rich Fibrin (PRF) as a socket graft material to enhance bone regeneration after tooth extraction and in a trial to minimize the alveolar ridge collapse prior to placement of dental implant placed in the maxilla. This study included 15 females (12 females and 3 males) healthy patients, they were free from any systemic disease or disorders that could affect the healing of bone. The patients were selected from the outpatient clinic of Oral and Maxillofacial Surgery Department; Faculty of Oral \& Dental Medicine, Cairo University. The patient's age ranged from 20 to 40 years. The need for extraction of unrepairable maxillary posterior teeth was determined by clinical and radiographic examination. The xenogenic cortico-cancellous bone graft was dispensed in a ready made syringe in putty form. The platelet rich fibrin preparation requires an adequate centrifuge, needle and $10 \mathrm{ml}$ blood collection tubes. The whole blood is drawn into the tubes without anticoagulant (as bovine thrombin) and is immediately centrifuged at $3000 \mathrm{rpm}$ for 10 minutes. The result is a fibrin clot containing the platelets located in the middle of the tube, just between the red blood cell layer at the bottom and a cellular plasma at the top. Teeth were extracted atraumatically, by using a surgical bur to separate the roots of the choosable maxillary posterior teeth. Each root was removed separately. Then the sockets were debrided and irrigated with sterile saline solution. Immediately after extraction of teeth, the teeth sockets were grafted with a combination of xenogenic cortico - cancellous bone graft and plateletrich fibrin and sutured with figure 8 sutures. The radiographic follow up intervals was performed using digital panoramic radiography, done after extraction of the tooth immediately at baseline
\end{abstract}

\footnotetext{
* Faculty of Oral \& Dental Medicine, Misr University for science and technology, B.D.S (2004)

** Professor of Oral and Maxillofacial Surgery, Faculty of Oral and Dental Medicine, Cairo University *** Lecturer of Oral and Maxillofacial Surgery, Faculty of Oral and Dental Medicine, Cairo University **** Lecturer of Oral and Maxillofacial Radiology, Faculty of Oral and Dental Medicine, Cairo University
} 
(0 month), 1 ,3, 4 and 6 months postoperatively. The statistical results revealed a statistically non-significant difference in mean bone density postoperatively from $0-1$ month observation periods, were followed by a statistically significant increase in mean bone density from $1-3$ months observation periods postoperatively. From $3-4$ months observation periods, there was non-significant statistically increase in mean bone density postoperatively. Comparing 0 month observation period with 4 months observation periods, there was statistically significant increase in mean bone density, also from $0-4$ months observation periods, there was statistically significant increase in mean values of bone density postoperatively as well as Ib group showed high density than control group, at 6 months period. Concerning the bone height, the statistical analysis showed that there was non-significant statistical difference between all intervals regarding the alveolar ridge height, through all periods in the study groups Ia and Ib. The histological results revealed that after 4 months from grafting. Several bony trabeculae were present having intercommunicating bone marrow spaces were clearly observed, the bone formed had a well trabeculated pattern and haversian system, with few amount of fibrous tissue. The viable osteocytes were randomly distributed through the bony trabeculae, which are an indicative of an area of new bone formation. The results of the current study histologically \& radiographically showed that the use of corticocancellous xenogenic graft in combination with PRF showed complete preservation of tooth socket dimensions. The density and quality of the alveolar ridge following bone regeneration after grafting with xenogenic graft + PRF were also determined by CBCT. Also; CBCT was used successfully in assessing preimplant insertion. Cone-beam computed tomography is providing valuable information to the recent study.

KEY WORD: Platelet rich fibrin, Xenogenic corticocancellous bone graft, Socket graft, bone regeneration after tooth extraction, alveolar ridge, collapse before implant placement

\section{INTRODUCTION}

Teeth loss is among the health problems associated with advanced age. Tooth loss necessitates prosthetic treatment for the patient to obtain the ability of proper function, to chew and eat as normally as possible. Since the physiological masticatory forces applied via the roots of teeth to the cancellous bone no longer persist. Tooth extraction is performed for a wide variety of reasons, such as tooth decay that may destroy enough tooth structure to prevent restorations, or periodontal disease, which may severely affect the tooth supporting tissues.

The dimensions of the alveolar bone are of great importance from both esthetic and implant planning reasons. Often when planning implant therapy, the socket grafting procedure is becoming a more necessary treatment step in order to restore adequate bone volume for implant insertion. Platelet rich fibrin (PRF) is a second generation platelet concentrate widely used to accelerate soft and hard tissue healing. Platelet rich fibrin (PRF) is a strictly autologous fibrin matrix containing a large quantity of platelet and leukocyte cytokines. It would be expected that PRF grafting for an extraction socket might result in enhanced wound healing. This will be the aim of the current study.

\section{REVIEW OF LITERATURE}

Platelet-rich fibrin (PRF) belongs to a new generation of platelet concentrates, with simplified processing and without biochemical blood handling. Here, blood is collected without any anticoagulant and immediately centrifuged. A natural coagulation process then occurs and allows for the easy collection of a leucocyte and platelet-rich fibrin (L-PRF) clot, without the need for any biochemical modification of the blood, that is, no anticoagulants, thrombin or calcium chloride are required. ${ }^{(1)}$ 
Platelet-rich fibrin (PRF) is one of the great challenges of clinical research has been the development of bioactive surgical additives, which help to regulate inflammation and increase the speed of healing process. The healing of hard and soft tissues both, is mediated by a wide range of intra and extra-articular events, which in turn are regulated by various signalling proteins. Understanding of this entire process is still not complete; however, it is known that platelets play a crucial role not only in haemostasis, but also in the wound healing process. ${ }^{(2-4)}$

\section{PATIENTS AND METHODS}

\section{I) Design of the clinical trials:}

Patients Selection: Fifteen patients with unrepairable maxillary posterior teeth were randomly selected from the out-patient clinic, of Oral and Maxillofacial Surgery Department Faculty of Oral and Dental Medicine, Cairo University.

\section{Patient Grouping:}

Fifteen eligible candidates were randomly divided into three main groups

\section{- In Group I:}

A study group which was subdivided into Ia and Ib

\section{- In Group Ia:}

Six endosseous implants were inserted in 6 patients at 4 months period from tooth socket grafting, with a mixture of cortico-cancellous xenogenic bone and platelet rich fibrin.

\section{- In Group Ib:}

Six endosseous implants were inserted in six patients at 6 months period from tooth socket grafting with the same mixture (cortico-cancelleous xenogenic bone graft +PRF)

\section{- In Group II:}

Control group in which 3 endosseous implants were inserted in 3 patients without grafting the tooth socket at 6 months period.

\section{II) Radiographic Examination:}

Pre-operative panoramic radiographs were taken to detect any pathosis (impacted or supernumerary teeth). radiographic data were reported for each patient.

\section{III) Preparation of the materials used}

\section{A-Xenogeneic Cortico Cancelleous bone graft:}

The graft material was (Cortico -Cancelleous Bone Xenograft). Osteobiol which is commercially available in sterilized sealed plastic syringe in putty form, the syringe containing $0.5 \mathrm{cc}$ of xenograft .The content of the syringe was injected directly into fresh extracted socket.

\section{B-Platelet Rich Fibrin (PRF) Preparation:}

According to Dohan Ehrenfest $2010^{(1)}$ platelet rich fibrin was prepared from patient's own blood before starting with implantation.

Platelet rich fibrin is defined as autologous leukocyte- and platelet rich fibrin biomaterials. Twenty c.c. of blood is collected in the sterile vacutainer tubes without anticoagulant. The vacutainer tubes are then placed in a centrifugal machine at 3000 revolutions per minute $(\mathrm{r} / \mathrm{m})$ for 10 minutes, after which it settles into the following three layers: red lower fraction containing red blood cells, upper straw coloured cellular plasma and the middle fraction containing the fibrin clot. The upper straw coloured layer is then removed and middle fraction is collected, $2 \mathrm{~mm}$ below lower dividing line, which is the PRF. A natural coagulation process then occurs and allows for the easy collection of a leucocytes- and platelet-rich fibrin (L-PRF) clot, without the need for any biochemical modification of the blood, that is, no anticoagulants, thrombin or calcium chloride are required. Platelet rich fibrin is simply considered as a natural optimized blood clot. 


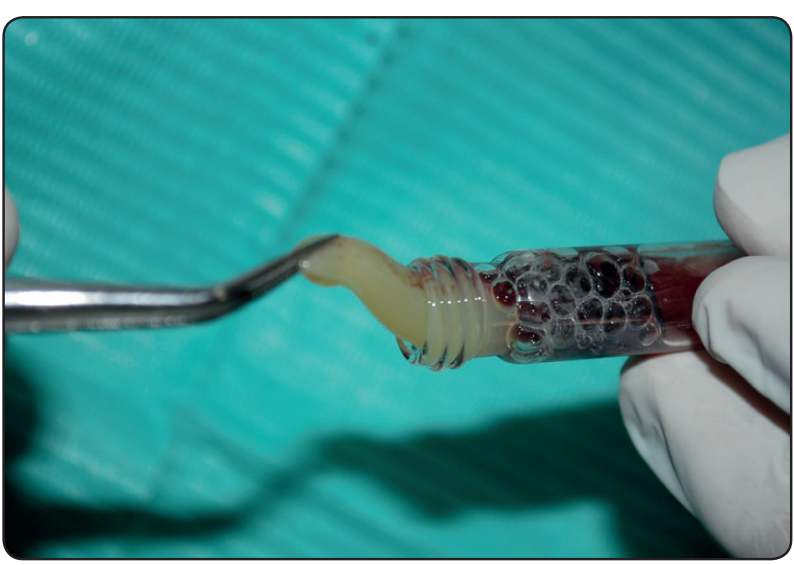

Fig. (1) Photographs showing of isolated Platelet-Rich Fibrin withdrawn from test tube

\section{C- Dental Implants:}

Fifteen endosseous implants IBS (S-Clean taper type) were inserted in the jaw using the same type of step drills.

IBS Dental implant is 2 stage implants are provided. The body is tapered evenly down from the top, which facilitate insertion in an undersized socket. The lengths of the implant used were between 12 to $14 \mathrm{~mm}$ and diameters between 3.7 to $5.8 \mathrm{~mm}$.

\section{IV- Surgical Approaches:}

\section{A-Phase 1 :}

Extraction of the maxillary posterior teeth.

A traumatic extraction to preserve the socket walls was done for each tooth and irrigation with saline $10 \% \mathrm{H}_{2} \mathrm{O}_{2}$ in a ratio of $3: 1$ followed by instillation of cortico-cancellous bone graft + PRF .

Instillation of mix two of xenogenic corticocancellous bone graft + Platelet Rich Fibrin were applied inside the socket.

\section{B-Phase 2 :}

Core biopsy before implant insertion was taken according to the scheduled time (group Ia after 4 months and group Ib after 6 months) Trephine drills
( $3 \mathrm{~mm}$ in diameter), same size of the implant drills were used to obtain core biopsies from the center of the grafted area, at a suitable speed (1500 rmp) with copious saline irrigation, containers were fixed with $2 \%$ formaldehyde solution for preservation of the bone core biopsy and sent for histological examination.

\section{Insertion of dental implants:}

A sequence of drilling started from 2.8 up to 5.8 according to the needed diameter for every tooth.

The implants were inserted at the grafted sockets. Cover screw was applied to cover the implant and sutured with 3-0 black silk sutures.

\section{Follow up and Evaluation Criteria:}

All patients will be subjected to clinical assessment to assess the soft tissue healing following extraction of teeth in each patient. Radiographic assessment will be performed using and cone beam CT (OPG) to assess bone density clinical evaluation at the following intervals:

- Immediately after extraction of teeth.

- One month post-extraction.

- 4 months postoperative.

- 6 months postoperative.

- Postimplant installation.

The collected data were tabulated and statistically analyzed.

\section{RESULTS}

This study was conducted on 15 female and 3 male healthy patients, aged from 20 to 40 years, who were required tooth extraction before implant placement. Patients had been selected from the outpatient clinic of Oral and Maxillofacial Surgery Department; Faculty of Oral \& Dental Medicine, Cairo University. 


\section{Clinical Findings:}

Clinically, all patients complied well with all study-related procedures. All patients were evaluated regarding the 15 extraction sockets wounds healed uneventfully \& the soft tissue healing proceeded normally as well, after grafting with a mix of xenogenic- corticocancellous and platelet rich fibrin, with no infection or any adverse reactions.

The platelet rich fibrin membrane was easy to handle and did not mutilated when manipulated. No complications or adverse reactions were reported regarding the healing following grafting with autologous platelet rich fibrin grafts. Follow-up visits were also scheduled at one, 4 and 6 months after grafting. The healing was evaluated and recorded immediately and after 4- 6 months by Cone Beam CT. by the end of the study period, the overall efficacy outcomes of the graft were promising \& the healing rate of the osseous defects was uneventful. The grafted sites (post-extraction sites) were reentered for implant placement after four months from grafting.

\section{Histological Observations}

Corebiopsies were taken from the regenerated areas in group Ia for histological analysis. The histological results revealed after 4 months from grafting by cortico-cancellous bone graft + platelet rich fibrin (PRF) that the maxillary molar extraction sockets had a satisfactory bone volume that filled of the osseous sockets.

The histological data revealed that after 4 months of fibrin (PRF) grafting, the tooth socket defects were occupied by newly formed bone. Active bone remodeling was demonstrated by the appearance of active osteogenesis, several bony trabeculation intercommunicating with bone marrow spaces that is filled with red type bone marrow.

Normal bone formation is seen in group II (control group) the specimens taken with large amount of bony

\section{Case from Study Group Ia}

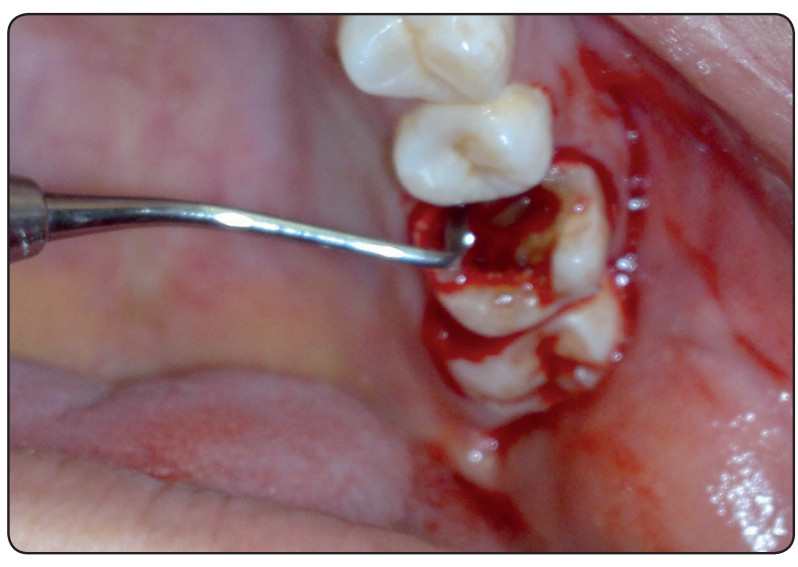

Fig.(2) Clinical photograph of study group Ia showing a traumatic extraction using periotome

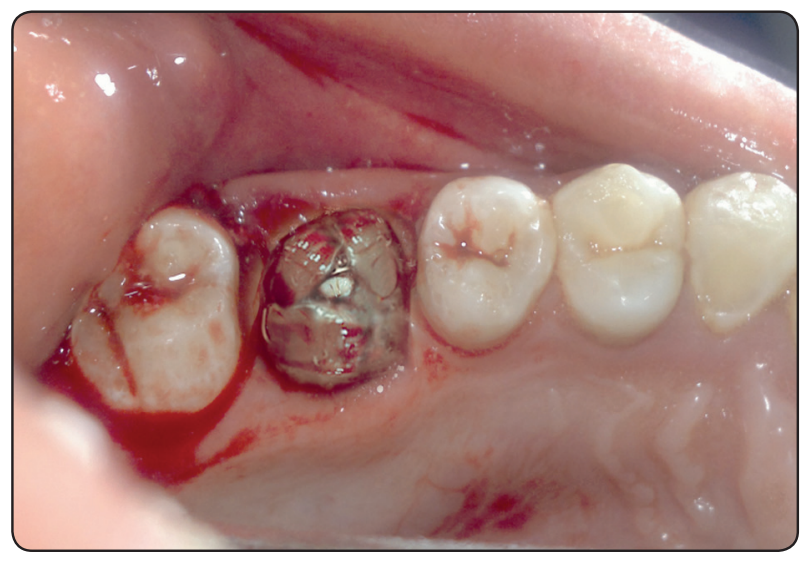

Fig.(3) Clinical photograph showing corticocancellous bone graft plus L-PRF all harvested together inside the socket of group Ia

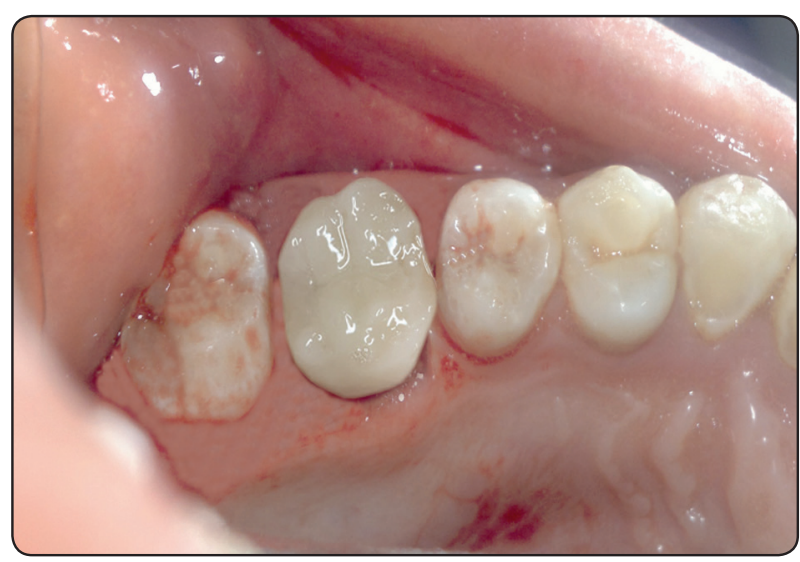

Fig.(4) Clinical photograph of study group Ia showing final restoration. 
trabeculations, the area of new bone formation was clearly observed, with few amounts of fibrous tissue. The fibrous tissue infiltrated with inflammatory cells.

The osteocytes were also shown inside their lacunae, which is indicative of an area of new bone formation, which were randomly distributed through the bony trabeculae.

Resting and reversal lines were marked in the newly formed bony trabeculae revealing a normal process of bone remodeling.

The histological results of this study showed that the placement of bone graft enhanced bone healing within a short time (4 months of grafting), compared to the normal period of new bone formation (6 months) period, as the area of bone formation was clearly observed.

Clinically, the placement of implants into the peri-implant sites after grafting with combination of xenogenic cortico-cancellous bone graft + PRF has shown high stability and success rates of implant placement.

\section{Radiographic follow up:}

Postoperative radiographic evaluations were achieved using CBCT scanning immediately postoperative (for study and control groups), 4 months post-operative (for study groub Ia), at 6 months post-operative (for study group Ib), and at 6 months postoperative(for the control group) to show bone changes in height and density. Measurements of alveolar bone height and density were taken from coronal and bucco-lingual cuts respectively.

\section{I-Descriptive findings}

\section{Study group Ia}

Immediate post-operative CBCT images revealed extraction sockets sharply outlined and filled with tissue density that is almost equivalent to the shadow of the gingival margin.
At four months post-operative, bone trabeculation was seen at the extraction site with higher density than normal surrounding bone, the socket boundaries were seen fading away, and the radio-opaque outline of crestal bone was gained.

\section{Study group $I b$}

Immediate post-operative CBCT images revealed extraction sockets sharply outlined and filled with tissue density that was almost equivalent to the shadow of the gingival margin.

At six months post-operative, the extraction socket can't be detected and the graft material could not be differentiated from the surrounding normal bone density. The radio-opaque outline of the crestal bone was regained.

\section{Control group:}

Immediate post-operative CBCT images revealed extraction sockets sharply outlined and filled with tissue density of hematoma that was almost equivalent to the shadow of the gingival margin.

At six months post-operative, the extraction socket can't be detected. The normal trabecular bone pattern and the crestal bone were gained.

\section{Statistical Analysis}

\section{A) Bone height:}

\section{Overall measurement (Mean of the four sides)}

Immediately post-operative, all the measurements were zero so no comparisons could be done.

At the follow up (4 months for group 1 and 6 months for group 2), there was no statistically significant difference between Study group 1(a) and Study group 1(b); both showed statistically significantly higher mean bone height measurements than control group. 


\section{B) Bone density:}

\section{Comparison between the three groups:}

Immediately post-operative, there was no statistically significant difference between the three groups.

At the follow up (4 months for group 1 and 6 months for group 2), there was no statistically significant difference between Study group 1(a) and Study group 1(b); both showed statistically significantly higher mean bone density than control group.

Table (6): Mean \pm standard deviation (SD) values and results of one-way ANOVA and Tukey's tests for the comparison between bone density measurements

\begin{tabular}{|c|c|c|c|c|}
\hline Time & Control & $\begin{array}{c}\text { Study } \\
\text { group 1 } \\
\text { (a) }\end{array}$ & $\begin{array}{c}\text { Study } \\
\text { group 1 } \\
\text { (b) }\end{array}$ & $P$-value \\
\hline $\begin{array}{c}\text { Immediate } \\
\text { post- } \\
\text { operative }\end{array}$ & $87.2 \pm 10.2$ & $81.5 \pm 9.2$ & $\begin{array}{c}88.1 \pm \\
11.6\end{array}$ & 0.175 \\
\hline Follow up & $93.5 \pm 9.4^{\mathrm{b}}$ & $\begin{array}{c}115.6 \pm \\
15.1^{\mathrm{a}}\end{array}$ & $\begin{array}{c}121.3 \pm \\
10.2^{\mathrm{a}}\end{array}$ & $0.006^{*}$ \\
\hline
\end{tabular}

*: Significant at $P \leq 0.05$, Different superscripts are statistically significantly different

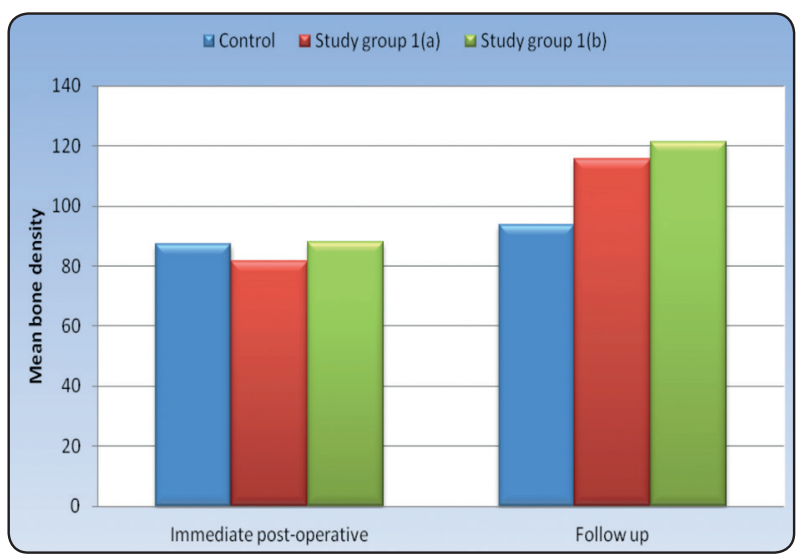

Fig. (5) Bar chart representing mean bone density in the three groups

\section{DISCUSSION}

The recent findings of group (Ia) showed statistically significantly increase in bone density. The difference in bone density between the two groups (Ia \& Ib/ control group) was about $16 \%$ in favor of group (I) i.e with in 4-6 months period. This is attributed to the growth factor of PRF, which is not present in the control group. Similarly, the growth factor according to Dohan 2010 ${ }^{(5)}$ was a contributing factor for growth of osteoblastic activity in absolutely building bone process which coincide with the histological findings of the core biopsy before insertion of the endosseous implants in the recent study.

The results of the current study histologically \& radiographically showed that the use of corticocancellous xenogenic graft in combination with Platelet Rich Fibrin showed almost complete preservation of tooth socket dimensions.

\section{SUMMARY}

The dimensions of the alveolar bone are of great importance from both esthetic and implant planning reasons. The preservation of bone volume immediately after tooth removal might be necessary to optimize the success of implant placement in terms of esthetics and function.

This study was conducted to investigate histologically, clinically \& radiographically, the potential of combination of xenogenic cortico - cancellous bone graft and platelet Rich Fibrin (PRF) as a socket graft material to enhance bone regeneration after tooth extraction and in a trial to minimize the alveolar ridge collapse prior to placement of dental implant placed in the maxilla.

This study included 15 females (12 females and 3 males) healthy patients, they were free from any systemic disease or disorders that could affect the healing of bone. The patients were selected from the outpatient clinic of Oral and Maxillofacial Surgery Department; Faculty of Oral \& Dental Medicine, Cairo University. 
The patient's age ranged from 20 to 40 years. The need for extraction of unrepairable maxillary posterior teeth was determined by clinical and radiographic examination.

The xenogenic cortico-cancellous bone graft was dispensed in a ready made syringe in putty form.

The platelet rich fibrin preparation requires an adequate centrifuge, needle and $10 \mathrm{ml}$ blood collection tubes. The whole blood is drawn into the tubes without anticoagulant (as bovine thrombin) and is immediately centrifuged at $3000 \mathrm{rpm}$ for 10minutes. The result is a fibrin clot containing the platelets located in the middle of the tube, just between the red blood cell layer at the bottom and a cellular plasma at the top.

Teeth were extracted atraumatically, by using a surgical bur to separate the roots of the choosable maxillary posterior teeth. Each root was removed separately. Then the sockets were debrided and irrigated with sterile saline solution. Immediately after extraction of teeth, the teeth sockets were grafted with a combination of xenogenic cortico cancellous bone graft and platelet-rich fibrin and sutured with figure 8 sutures.

The radiographic follow up intervals was performed using digital panoramic radiography, done after extraction of the tooth immediately at baseline (0 month), $1,3,4$ and 6 months postoperatively.

The statistical results revealed a statistically non-significant difference in mean bone density postoperatively from $0-1$ month observation periods, were followed by a statistically significant increase in mean bone density from $1-3$ months observation periods postoperatively. From 3 4 months observation periods, there was nonsignificant statistically increase in mean bone density postoperatively. Comparing 0 month observation period with 4 months observation periods, there was statistically significant increase in mean bone density, also from $0-4$ months observation periods, there was statistically significant increase in mean values of bone density postoperatively as well as $\mathrm{Ib}$ group showed high density than control group, at 6 months period.

Concerning the bone height, the statistical analysis showed that there was non-significant statistical difference between all intervals regarding the alveolar ridge height, through all periods in the study groups Ia and Ib.

The histological results revealed that after 4 months from grafting. Several bony trabeculae were present having intercommunicating bone marrow spaces were clearly observed, the bone formed had a well trabeculated pattern and haversian system, with few amount of fibrous tissue. The viable osteocytes were randomly distributed through the bony trabeculae, which are an indicative of an area of new bone formation.

The results of the current study histologically \& radiographically showed that the use of corticocancellous xenogenic graft in combination with PRF showed complete preservation of tooth socket dimensions.

The density and quality of the alveolar ridge following bone regeneration after grafting with xenogenic graft + PRF were also determined by CBCT. Also; CBCT was used successfully in assessing pre-implant insertion. Cone-beam computed tomography is providing valuable information to the recent study.

\section{CONCLUSION}

- The combination of xenogenic cortico cancellous bone graft of platelet rich fibrin enhanced bone formation within a short time (4 months of grafting), compared to the normal period of new bone formation ( 6 months) period. 
- The above mentioned combination graft have reported synergetic effects on healing processes as a socket preservation.

- The graft combination was easy to handle, biocompatible and well tolerated by the host.

- The utilization of cone beam computed tomography and digital panoramas has shown a great promise as an adjunct tool for diagnosis and follow ups.

\section{RECOMMENDATIONS}

- Further investigations are recommended on a large scale of patients plus long term follow up.

- It is advocated to use Cortico-Cancellous xenogenic bone grafts with PRF as a potential material for filling other osseous defects related to the maxillofacial areas.

\section{REFERENCES}

1. Simonpieri A, Choukroun J, Girard MO, Ouaknine T, Dohan D. [Immediate post-extraction implantation: interest of the PRF.].

2. Dohan DM, Choukroun J, Diss A, Dohan SL, Dohan AJ, Mouhyi J, et al. Platelet-rich fibrin (PRF): A secondgeneration platelet concentrate, Part I: Technological concepts and evolution. Oral Surg Oral Med Oral Pathol Oral Radiol Endod 2006;101:E37-44.

3. Gabling VL, Acil Y, Springer IN, Hubert N, Wiltfang J. Platelet-rich plasma and Platelet-rich fibrin in human cell culture. Oral Surg Oral Med Oral Pathol Oral Radiol Endod 2009;108:48-55.

4. Anitua E, Andia I, Ardanza B, Nurden P, Nurden AT. Autologous platelets as a source of proteins for healing and tissue regeneration. Thromb Haemost 2004; 91: 4-15

5. Dohan Ehrenfest DM, Coelho PG, Kang BS, Sul YT, Albrektsson T. Classification of osseointegrated implant surfaces: materials, chemistry and topography. Trends Biotechnol. 2010; 28:198-206. 